\title{
Stochastic Admission Control for Quality of Service in Wireless Packet Networks
}

\author{
Majid Ghaderi ${ }^{1}$, Raouf Boutaba ${ }^{1}$, and Gary W. Kenward ${ }^{2}$ \\ 1 University of Waterloo, Waterloo, ON N2L 3G1, Canada \\ \{mghaderi, rboutaba\}@uwaterloo.ca \\ 2 Nortel Networks, Ottawa, ON K1Y 4H7, Canada \\ gkenward@nortelnetworks.com
}

\begin{abstract}
Call admission control is a key element for providing quality of service (QoS) in mobile wireless networks. Traditional admission control schemes only address call-level QoS guarantee because of the underlying circuit-based network architecture. In contrast, emerging wireless technologies such as $3 \mathrm{G}$ and $4 \mathrm{G}$ tend to be packet-switched rather than circuit-switched because the packet-based architecture allows better sharing of the scarce wireless resources. This paper introduces a novel distributed call admission control scheme called PFG, which maximizes the wireless channel utilization subject to a predetermined bound on the call dropping and packet loss probabilities for variable-bit-rate traffic in a packet-switched wireless cellular network. In particular, we show that in wireless packet networks, the undesired event of dropping an ongoing call can be completely eliminated without sacrificing the bandwidth utilization. Extensive simulation results show that our scheme satisfies the hard constraint on call dropping and packet loss probabilities while maintaining a high bandwidth utilization.
\end{abstract}

\section{Introduction}

Emerging wireless technologies such as 3G and 4G [1, 2] tend to be packetswitched rather than circuit-switched because the packet-based architecture allows better sharing of limited wireless resources. In a packet network, calls do not require dedicated circuits for the entire duration of connection. Unfortunately, this enhanced flexibility makes it more difficult to effectively control the admission of connections into the network.

In wireless packet networks there exist two levels of quality of service, namely, call-level and packet-level. At call-level, two important parameters which determine the quality of service are call blocking probability and call dropping probability. Since dropping a call in progress has more negative impact from the user perspective, handoff calls are given higher priority than new calls in access to wireless resources. At packet-level, packet loss probability, delay and jitter are the most important QoS parameters. There is always a trade-off between the network utilization and the QoS perceived by users. It is desired to have a re- 
source allocation scheme which can satisfy the prespecified QoS constraints while maximizing the utilization of the network resources.

Call admission control (CAC) has been extensively studied in circuit-switched (voice) wireless cellular networks (see [3, 4, 5] and references there in). Hong and Rappaport [6] are the first who systematically analyzed the famous guard channel (GC) scheme, which is currently deployed in cellular networks supporting voice calls. Ramjee et al. [7] showed that the guard channel scheme is optimal for minimizing a linear objective function of call blocking and dropping probabilities while the fractional guard channel scheme (FG) is optimal for minimizing call blocking probability subject to a hard constraint on call dropping probability. Instead of explicit bandwidth reservation as in the GC, the FG accepts new calls according to a randomization parameter called the acceptance ratio. Because of user mobility, it is impossible to describe the state of the system by using only local information, unless we assume that the network is uniform and approximate the overall state of the system by the state of a single cell in isolation. To include the global effect of mobility, collaborative or distributed admission control schemes have been proposed [8, 9, 10, Information exchange among a cluster of neighboring cells is the approach adopted by all distributed schemes.

None of these papers has considered a wireless packet-switched network. There is no packet-level consideration in these works. Call dropping and blocking probabilities are the only QoS parameters considered. In circuit-switched networks, when a handoff call arrives while there is no idle circuit (wireless channel), the handoff fails and hence the call is dropped. In contrast, in a packet-switched network it is still possible to accept the handoff call at the expense of probably increasing the number of dropped packets. While this approach completely eliminates the call dropping event, we will show that its impact on packet loss can be effectively controlled.

We introduce a packetized fractional guard channel (PFG) call admission control mechanism for cellular packet networks that achieves a high bandwidth utilization while satisfying a target packet loss probability without dropping any ongoing call. To the best of our knowledge, PFG is the first to address the combination of call-level and packet-level QoS while explicitly considering the mobility of users. The main features of PFG are as follows:

1. PFG achieves zero percent call dropping,

2. $\mathrm{PFG}$ is dynamic, therefore, adapts to a wide range of system parameters and traffic conditions,

3. $\mathrm{PFG}$ is distributed and takes into consideration the information from direct neighboring cells in making admission decisions,

4. The control mechanism is stochastic and periodic to reduce the overhead associated with distributed control schemes.

The motivation behind this study is to support variable-bit-rate (VBR) multimedia traffic in emerging wireless packet cellular networks. The rest of the 
paper is organized as follows. Our system model, assumptions and notations are described in section 2. Section 3 describes the high-level operation of the proposed admission control algorithm while in section 4 , detailed analysis of the algorithm is presented. Simulation results are presented in section 5 and section 6] concludes this paper.

\section{System Model}

The considered system is a packet-switched cellular network, in which the users move along an arbitrary topology of $\mathcal{B}$ cells according to the routing probabilities $r_{i j}$ (from cell $i$ to cell $j$ ). Each cell $i$ has a set of adjacent cells denoted by $\mathcal{A}_{i}$. We assume that there is one type of calls in the system. Although the dynamic behavior of wireless channel may cause packets to be dropped, we assume that there are appropriate underlying coding and retransmission mechanisms to cope with packet loss due to channel effects. Therefore, cell overflow, receiving more traffic than what can be actually transmitted, is considered the only source of packet loss. This paper considers constant cell capacity, however, the approach that we propose next can be extended to include variable capacity cases using a technique similar to the one proposed in [11. Moreover, we assume that

1. New call arrivals to a cell are independent and Poisson distributed with rate $\lambda_{i}$.

2. Cell residency times are independent and exponentially distributed with mean $1 / h$. However, we show that the proposed algorithm is insensitive to this assumption.

3. Call durations are independent and exponentially distributed with mean $1 / \mu$.

These assumptions are widely used in literature $[4,5,3,6,7,8,9,10$, for the mean value analysis of cellular systems.

\subsection{Maximum Occupancy in a Cell}

Let $M_{i}$ denote the maximum occupancy, i.e. maximum number of calls, in cell $i$ under the so-called average bandwidth assignment scheme. This scheme allocates to each VBR call a share of bandwidth equal to the call's average bandwidth requirement. Let $m$ denote the average bandwidth requirement of a call, then

$$
M_{i}=\frac{c_{i}}{m}
$$

where $c_{i}$ denoted the capacity of cell $i$. Although this scheme achieves a high bandwidth utilization, it leads to a high rate of packet loss [12. If there are more than $M_{i}$ calls in cell $i$, then we say that the cell is in overloaded state. In the overloaded state, probability of packet loss is very high. Our scheme, PFG, rejects new call requests when a cell is in overloaded state. 


\section{$2.2 \quad$ Time-Dependent Handoff Probability}

Let $P_{h}(t)$ denote the probability that a call hands off to another cell by time $t$ and remains active until $t$, given that it has been active at time 0 . Also, let $P_{s}(t)$ denote the probability that a call remains active in its home cell until time $t$, given that it has been active at time 0 . It is obtained in $\left[13\right.$ that $P_{h}(t)=$ $\left(1-e^{-h t}\right) e^{-\mu t}$ and $P_{s}(t)=e^{-(\mu+h) t}$. On average, for any call which arrives at time $t^{\prime} \in(0, t]$, the average handoff and stay probabilities $\tilde{P}_{h}$ and $\tilde{P}_{s}$ are expressed as

$$
\begin{aligned}
& \tilde{P}_{h}(t)=\frac{1}{t} \int_{0}^{t} P_{h}\left(t-t^{\prime}\right) d t^{\prime}, \\
& \tilde{P}_{s}(t)=\frac{1}{t} \int_{0}^{t} P_{s}\left(t-t^{\prime}\right) d t^{\prime} .
\end{aligned}
$$

Similar to 8 and [9, we assume that during a control period each call experiences at most one handoff. This assumption is justified by setting the length of the control period $T$ reasonably shorter than the average cell residency time. Discussion on the appropriate control interval is not included here due to paper length restriction. For the optimal control interval $T$, please refer to [13].

Finally, let $P_{j i}(t)$ denote the time-dependent handoff probability that an active call in cell $j$ at time 0 will be in cell $i$ at time $t$, where $j \in \mathcal{A}_{i}$. Since each call experiences at most one handoff during the control period, it is obtained that $P_{j i}(t)=P_{h}(t) r_{j i}$. Similarly, the average handoff probability $\tilde{P}_{j i}(t)$ for a call which arrives at any time $t^{\prime} \in(0, t]$ is given by $\tilde{P}_{j i}(t)=\tilde{P}_{h}(t) r_{j i}$.

\section{Admission Control Algorithm}

The proposed distributed algorithm, PFG, consists of two components. The first component is responsible for retrieving the required information from the neighboring cells and computing the acceptance ratio. Using the computed acceptance ratio, the second component enforces the admission control locally in each cell. The following sections describe these two components in detail.

\subsection{Distributed Control Algorithm}

To reduce the signalling overhead all the information exchange and acceptance ratio computations happen only once at the beginning of each control period of length $T$. Several steps involved in PFG distributed control are described below:

1. At the beginning of a control period, each cell $i$ sends the number of active calls in the cell at the beginning of the control period denoted by $N_{i}(0)$ to its adjacents and receives the number of new calls, $N_{i}$, which were admitted in the last control period by each adjacent cell.

2. Now, cell $i$ uses the received information and those available locally to compute the acceptance ratio $a_{i}$ using the technique described in section 4

3. Finally, the computed acceptance ratio $a_{i}$ is used to admit call requests into cell $i$ using the algorithm presented in section 3.2 . 


\subsection{Local Control Algorithm}

Let $s_{i}$ denote the state of cell $i$, where there are $s$ calls active in the cell. Let $a_{i}(s)$ denote the acceptance ratio where the cell state is $s_{i}$. Fig. 11 shows the state transition diagram of the PFG scheme in cell $i$. In this diagram, $\nu_{i}$ is the handoff arrival rate into cell $i$, and $M_{i}$ is the maximum occupancy given by (11).

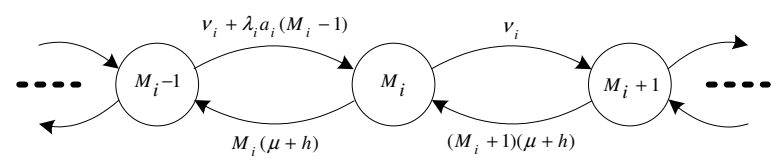

Fig. 1. Packetized fractional guard channel transition diagram

The pseudo-code for the local admission control in cell $i$ is given by the algorithm in Fig. 2. In this algorithm, $x$ is a call requesting a connection into cell $i$. The acceptance ratio for the respective control period is $a_{i}$. Also, $\operatorname{rand}(0,1)$ is the standard uniform random generator function. In the next section, we will present a technique to compute the acceptance ratio $a_{i}$ in order to complete this algorithm.

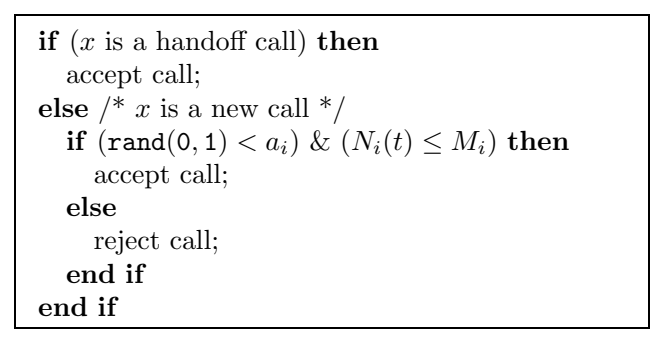

Fig. 2. Local call admission control algorithm in cell $i$

\section{Computing the Acceptance Ratio}

Assuming the target loss probability is sufficiently small, we approximate the packet loss probability by the overflow probability in each cell. This is often an overestimate of the actual buffer overflow probability since it ignores the smoothing effect of the buffer, i.e. the buffer allows the arrival rate to exceed the service rate for short periods. The significance of such inaccuracies must be tempered by the fact that even an exact model does not provide a correct measure of the loss probability seen by calls, as it can not fully capture the impact of interactions within the network. This is a common technique in approximating packet loss probability (see for example [12]). However, as the overflow probability decays to zero, both measures converge to the same value and the difference becomes 
negligible. Therefore, the time-dependent packet loss probability at time $t$ in cell $i$ denoted by $L_{i}(t)$ is given by

$$
L_{i}(t)=\operatorname{Pr}\left(R_{i}(t)>c_{i}\right),
$$

where $R_{i}(t)$ denotes the total (new and handoff) packet arrival rate into cell $i$ at time $t$. The main idea is to describe $R_{i}(t)$ using a Gaussian distribution. The motivation behind Gaussian traffic characterization is that it is very natural when a large number of traffic sources are multiplexed (motivated by the central limit theorem), as is expected to be the case in future wireless networks.

\subsection{Traffic Characterization}

Let $r_{n}$ denote the packet generating process of an individual call $n$. It is assumed that individual packet generating processes are independent and identically distributed (iid) random variables with the mean and variance $E[r]$ and $V[r]$, respectively. Then, $R_{i}(t)$, the total packet arrival rate in cell $i$ at time $t$, is expressed as

$$
R_{i}(t)=\sum_{n=1}^{N_{i}(t)} r_{n}
$$

where $N_{i}(t)$ denotes the number of calls at time $t$. To characterize $R_{i}(t)$ by a Gaussian distribution we need to specify the parameters of $R_{i}(t)$, namely, mean and variance. Using the moment generating functions of random processes $R_{i}(t)$ and $r_{n}$, it is obtained that (please refer to [13] for details)

$$
\begin{aligned}
E\left[R_{i}(t)\right] & =E\left[N_{i}(t)\right] E[r], \\
V\left[R_{i}(t)\right] & =E\left[N_{i}(t)\right] V[r]+V\left[N_{i}(t)\right] E^{2}[r] .
\end{aligned}
$$

Given that $E[r]$ and $V[r]$ are first order statistics, they can be estimated from measured traffic data which makes this traffic characterization ideal from a measurement point of view [14]. Also, individual packet generating processes can have arbitrary correlation structure and this includes self-similar processes as well.

\subsection{Mobility Characterization}

The number of calls in cell $i$ at time $t$ is affected by two factors: (1) the number of background (existing) calls which are already in cell $i$ or its adjacent cells, and, (2) the number of new calls which will arrive in cell $i$ and its adjacent cells during the period $(0, t](0<t \leq T)$. Let $g_{i}(t)$ and $n_{i}(t)$ denote the number of background and new calls in cell $i$ at time $t$, respectively.

A background call in cell $i$ will remain in cell $i$ with probability $P_{s}(t)$ or will handoff to an adjacent cell $j$ with probability $P_{i j}(t)$. A new call which is admitted in cell $i$ at time $t^{\prime} \in(0, t]$ will stay in cell $i$ with probability $\tilde{P}_{s}(t)$ or will handoff to an adjacent cell $j$ with probability $\tilde{P}_{i j}(t)$. Therefore, the number of background calls which remain in cell $i$ and the number of handoff calls which come into cell $i$ during the interval $(0, t]$ are binomially distributed. Using this 
property, the time-dependent variance of stay and handoff processes denoted by $V_{s}(t)$ and $V_{j i}(t)$ and their average counterparts denoted by $\tilde{V}_{s}(t)$ and $\tilde{V}_{j i}(t)$ can be computed (please refer to [13] for details).

The number of calls in cell $i$ is the summation of the number of background calls, $g_{i}(t)$, and new calls, $n_{i}(t)$. Therefore, the mean number of active calls in cell $i$ at time $t$ is given by

$$
E\left[N_{i}(t)\right]=E\left[g_{i}(t)\right]+E\left[n_{i}(t)\right]
$$

where,

$$
\begin{aligned}
& E\left[g_{i}(t)\right]=N_{i}(0) P_{s}(t)+\sum_{j \in \mathcal{A}_{i}} N_{j}(0) P_{j i}(t), \\
& E\left[n_{i}(t)\right]=\left(a_{i} \lambda_{i} t\right) \tilde{P}_{s}(t)+\sum_{j \in \mathcal{A}_{i}}\left(a_{j} \lambda_{j} t\right) \tilde{P}_{j i}(t) .
\end{aligned}
$$

Similarly the variance is given by

$$
V\left[N_{i}(t)\right]=V\left[g_{i}(t)\right]+V\left[n_{i}(t)\right]
$$

where,

$$
\begin{aligned}
V\left[g_{i}(t)\right] & =N_{i}(0) V_{s}(t)+\sum_{j \in \mathcal{A}_{i}} N_{j}(0) V_{j i}(t), \\
V\left[n_{i}(t)\right] & =\left(a_{i} \lambda_{i} t\right) \tilde{V}_{s}(t)+\sum_{j \in \mathcal{A}_{i}}\left(a_{j} \lambda_{j} t\right) \tilde{V}_{j i}(t) .
\end{aligned}
$$

Note that given the arrival rate $\lambda_{i}$ and the acceptance ratio $a_{i}$, the actual new call arrival rate into cell $i$ is given by $\bar{\lambda}_{i}=\lambda_{i} a_{i}$. Therefore, the expected number of call arrivals during the interval $(0, t]$ is given by $a_{i} \lambda_{i} t$. Instead of using the actual value of $\bar{\lambda}_{j}$ which is not known at the beginning of the new control interval (time 0 ), each cell $i$ estimates the actual new call arrival rates of its adjacents for the new control period using an exponentially weighted moving average technique, i.e. $\bar{\lambda}_{j} \leftarrow(1-\epsilon) \frac{N_{j}}{T}+\epsilon \bar{\lambda}_{j}$. In our simulations we found that $\epsilon=0.3$ leads to a good estimation of the actual new call arrival rate.

\subsection{Packet Loss Probability}

As mentioned earlier, the packet arrival distribution in each cell can be approximated by a Gaussian distribution:

$$
R_{i}(t) \sim \mathbf{G}\left(E\left[R_{i}(t)\right], V\left[R_{i}(t)\right]\right),
$$

where, $E\left[R_{i}(t)\right]$ and $V\left[R_{i}(t)\right]$ are given by (6) and (7), respectively. Using (4) and (14) it is obtained that

$$
L_{i}(t)=\frac{1}{2} \operatorname{erfc}\left(\frac{c_{i}-E\left[R_{i}(t)\right]}{\sqrt{2 V\left[R_{i}(t)\right]}}\right),
$$


where, $\operatorname{erfc}(c)=\frac{2}{\sqrt{\pi}} \int_{c}^{\infty} e^{-t^{2}} d t$. Using (15), the average packet loss probability over a control period of length $T$ is given by

$$
\tilde{L}_{i}=\frac{1}{T} \int_{0}^{T} L_{i}(t) d t
$$

Then, the acceptance ratio, $a_{i}$, can be found by numerically solving equation

$$
\tilde{L}_{i}=P_{L}
$$

where $P_{L}$ is the target packet loss probability. The boundary condition is that $a_{i} \in[0,1]$, hence if $\tilde{L}_{i}$ is less than $P_{L}$ even for $a_{i}=1$ then $a_{i}$ is set to 1 . Similarly, if $\tilde{L}_{i}$ is greater than $P_{L}$ even for $a_{i}=0$, then $a_{i}$ is set to 0 .

\section{$5 \quad$ Simulation Results}

\subsection{Simulation Parameters}

Simulations were performed on a two-dimensional cellular system consisting of 19 hexagonal cells where opposite sides wrap-around to eliminate the finite size effect. As the basic traffic type, packetized voice calls are generated for simulation purposes. For packetized voice, a packet loss probability of $P_{L}=0.01$ is acceptable. The common parameters used in the simulation are as follows. All the cells have the same capacity $c$. Target packet loss probability is $P_{L}=0.01$, control interval is set to $T=20 s$ and $r_{j i}=1 / 6$. In all of the cases simulated, normalized load $\rho=\frac{1}{M_{i}}\left(\frac{\lambda}{\mu}\right)$ is used, where $M_{i}$ is given by (11). For each load, simulations were done by averaging over 8 samples, each for $10^{4} s$ of simulation time. Unless otherwise mentioned, call duration and cell residency times are exponentially distributed with means $\mu^{-1}=180 \mathrm{~s}$ and $h^{-1}=100 \mathrm{~s}$, respectively. We found this set of parameters more or less common and reasonable for a simulation setup (see for example [10]).

A two-state Markov model is used to describe the traffic generation process of voice calls. In this model, $\alpha$ and $\beta$ are transition rates to $\mathrm{OFF}$ and $\mathrm{ON}$ states, respectively, from ON and OFF states. While in the ON state, traffic is generated at a constant rate of $A$ packet/sec. For this traffic model, the mean and variance of the traffic generated are given by $E[r]=\frac{\beta}{\alpha+\beta} A$ and $V[r]=\frac{\alpha \beta}{(\alpha+\beta)^{2}} A^{2}$. Commonly used parameters for human speech representation are $\alpha^{-1}=1.2 \mathrm{~s}$ and $\beta^{-1}=1.8 s$ [12. Using an $8 \mathrm{Kbps}$ encoded voice source, it is obtained that $A=100$ packet $/ \mathrm{sec}$ and hence, $E[r]=40$ packet/sec and $V[r]=50$ packet $/ \mathrm{sec}$ assuming that each packet is 80 bits.

\subsection{Conservative PFG}

As mentioned earlier, PFG does not drop any handoff call, instead some packets may be dropped to accommodate the incoming handoff packets. To have an intuition about the impact of accepting handoffs even during the overloaded 
state, we have also implemented a slightly different version of PFG in addition to the original PFG represented in Fig. 1. This modified version drops handoffs during the overloaded state. We refer to the original algorithm by PFG-D0 and the modified one by PFG-DP where D0 and DP stand for zero dropping probability and $P$ dropping probability, i.e. if we use PFG-DP instead of PFG-D0 then there will be $P$ percent call dropping. Our purpose is to find the value of $P$ for some simulated scenarios to see how far it is from zero. Notice that, having $N_{i}(t)>M_{i}(t \in(0, T])$ indicates that cell $i$ is in the overloaded state at time $t$.

\subsection{Results and Analysis}

As mentioned earlier, PFG is the first to achieve zero call dropping while guaranteeing a hard constraint on packet loss probability. To the best of our knowledge there is no existing scheme which takes into consideration a combination of call-level and packet-level QoS parameters while taking into consideration the mobility of users. Therefore, we are not able to compare the performance of PFG with any other scheme. Instead, by doing extensive simulations, we have shown that PFG can achieve its defined goals.

Effect of Cell Capacity: Intuitively, increasing the cell capacity leads to a better Gaussian approximation, and hence, a more accurate admission decision. To investigate the effect of cell capacity, we considered three different capacity configurations, namely, $c 1=1 \mathrm{Mbps}, c 2=2 \mathrm{Mbps}$ and $c 5=5 \mathrm{Mbps}$ (for $3 \mathrm{G}$ and $4 \mathrm{G}$ systems). Normalized loads in range $[0 \ldots 2]$ are simulated, where the normalized load is defined as mentioned before. In Figs. 3(a) and 3(b) we have circled a region around load $\rho=1.0$. This is the most interesting part of the system which is likely to happen in practice. In the following discussion we refer to this region as the operating region of the system.

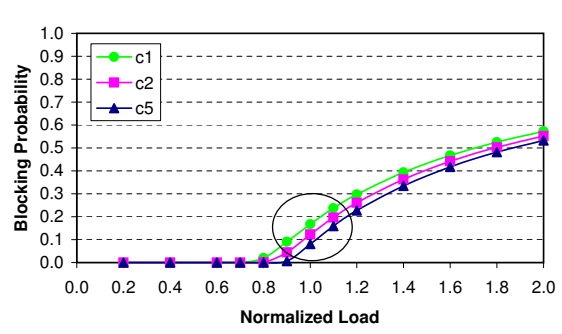

(a) Blocking probability

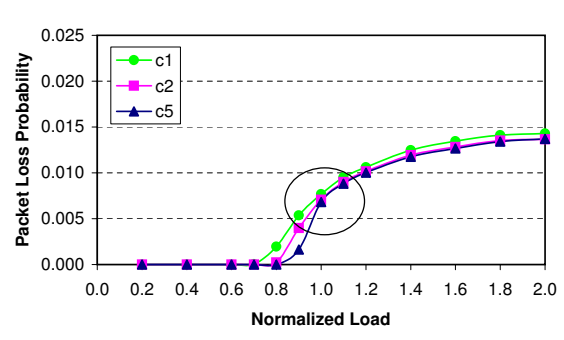

(b) Packet loss probability

Fig. 3. PFG-D0 performance results

Fig. 3(a) shows the new call blocking probability. It is clear from the figure that as the cell capacity increases the blocking probability decreases which can be explained from the central limit theorem and Gaussian approximation used in 
section 4.3. The packet loss probability, $\tilde{L}_{i}$, is depicted in Fig. $3(\mathrm{~b})$ Although $\tilde{L}_{i}$ goes beyond the target limit for high system loads, it is completely satisfactory for the operating region. Nevertheless, it is quite possible to modify PFG-D0 in order to make it more conservative for high loads. Similar to call blocking, as the capacity increases the PFG-D0 efficiency improves. After all, $c 1$ produces rather accurate results and increasing the capacity beyond it produces only marginal improvements.

Effect of Accepting Handoffs in Overloaded State: To investigate the impact of accepting handoffs during the overloaded state (in which $N_{i}(t)>M_{i}$ ), we ran PFG-DP for the same simulation configuration we ran PFG-D0. It was observed that the call dropping probability is almost zero in all the simulated configurations (please refer to [13] for detailed numerical results). It means that basically there is no difference between two schemes in terms of the call dropping probability. Fig. 4 shows the call blocking and packet loss probabilities of PFGD0 versus PFG-DP when the system capacity is set to $c 1$ (1 Mbps). It can be seen from Figs. 4(b) and Fig. 4(a) that the call blocking probability and packet loss probability are almost the same for both schemes indicating that accepting handoffs during the overloaded state has a negligible effect on the admission control performance.

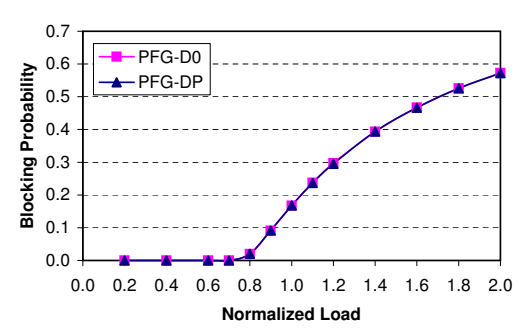

(a) Blocking probability

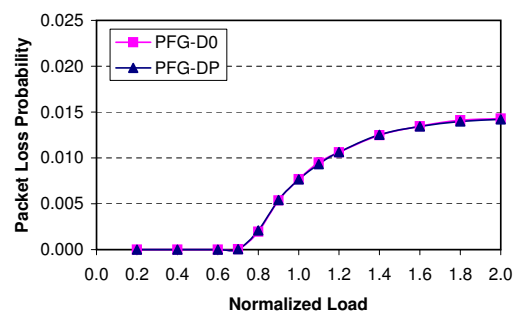

(b) Packet loss probability

Fig. 4. PFG-D0 vs. PFG-DP

Effect of Mobility: Define the mobility factor to be $\alpha=h / \mu$. Intuitively, $\alpha$ shows the average number of handoff attempts a call makes during its life time. As the mobility factor increases the handoff arrival rate increases as well. To investigate the impact of mobility on PFG, we have simulated three mobility cases, namely, (Mob:high, $\alpha=9.00$ ), (Mob:mod, $\alpha=1.80$ ) and (Mob:low, $\alpha=$ 0.36 ) for the base capacity $c 1$. Observed from Fig. 5. PFG is almost insensitive to the mobility rate of users. As shown in Figs. 5(a) the call blocking probability is almost match. Furthermore, Fig. 5(b) shows that the effect of mobility on packet loss probability is not very significant. In all three cases, PFG is able to satisfy the target packet loss probability in the operating region of the system. In general, handoff degrades the performance of cellular systems. 


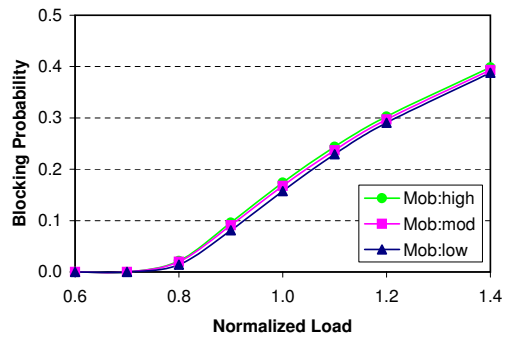

(a) Blocking probability

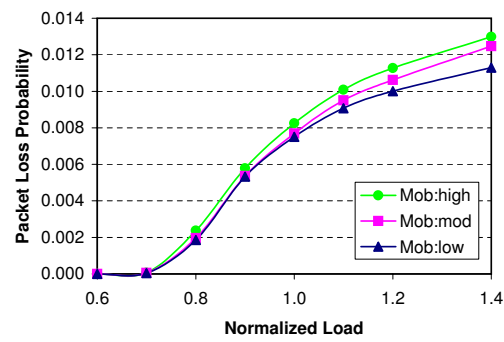

(b) Packet loss probability

Fig. 5. Mobility impact on PFG-D0 performance

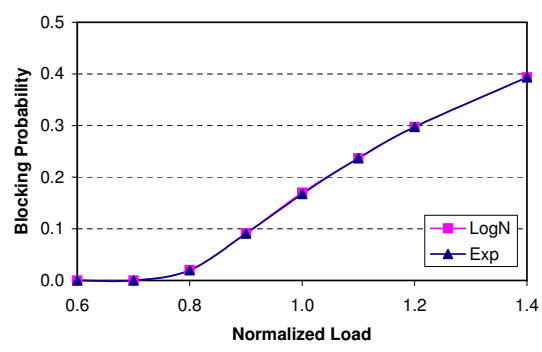

(a) Blocking probability

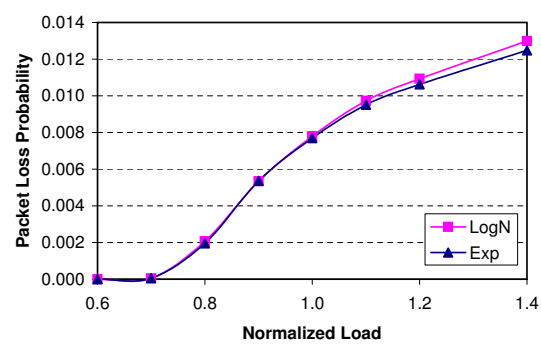

(b) Packet loss probability

Fig. 6. Lognormal vs. Exponential residence time

Effect of Non-exponential Cell Residence Times: Using real measurements, Jedrzycki and Leung [15] showed that a lognormal distribution is a more accurate model for cell residency time than the exponential distribution. Fig. [6 shows the call blocking and packet loss probability of exponential cell residency versus lognormal cell residency with the same mean and variance. It is observed that the exponential cell residency achieves sufficiently accurate control. In other words, the control algorithm is rather insensitive to this assumption due to its periodic control in which the length of the control interval is much smaller than the mean residency time.

\section{Conclusion}

In this paper we presented a novel scheme for admission control and hence QoS provisioning for packet-switched cellular systems. In essence, our approach is the natural generalization of the well-known effective bandwidth concept proposed for wireline networks. Through analysis and simulation, we showed that the proposed scheme, PFG, is able not only to improve utilization of scarce wireless 
bandwidth thanks to the statistical multiplexing of VBR traffic sources but also to eliminate the undesirable call dropping event inherent to circuit-switched cellular systems. In wireless multimedia networks, there are different service classes, each of which has its own packet and call level QoS constraints. We are currently investigating the extension of PFG to multiple service classes where each service class has its own QoS requirements.

\section{References}

1. Zahariadis, T., Kazakos, D.: (R)evolution toward $4 \mathrm{G}$ mobile communication systems. IEEE Wireless Commun. Mag. 10 (2003) 6-7

2. Hui, S.Y., Yeung, K.H.: Challenges in the migration to $4 \mathrm{G}$ mobile systems. IEEE Computer 41 (2003) 54-59

3. Peha, J.M., Sutivong, A.: Admission control algorithms for cellular systems. ACM/Kluwer Wireless Networks 7 (2001) 117-125

4. Fang, Y., Zhang, Y.: Call admission control schemes and performance analysis in wireless mobile networks. IEEE Trans. Veh. Technol. 51 (2002) 371-382

5. Gao, Q., Acampora, A.: Performance comparisons of admission control strategies for future wireless networks. In: Proc. IEEE WCNC'02. Volume 1., Orlando, USA (2002) 317-321

6. Hong, D., Rappaport, S.S.: Traffic model and performance analysis for cellular mobile radio telephone systems with prioritized and nonprioritized handoff procedures. IEEE Trans. Veh. Technol. 35 (1986) 77-92 See also: CEAS Tech. Rep. No. 773, College of Engineering and Applied Sciences, State University of New York, June 1999.

7. Ramjee, R., Towsley, D., Nagarajan, R.: On optimal call admission control in cellular networks. ACM/Kluwer Wireless Networks 3 (1997) 29-41

8. Naghshineh, M., Schwartz, M.: Distributed call admission control in mobile/wireless networks. IEEE J. Select. Areas Commun. 14 (1996) 711-717

9. Epstein, B.M., Schwartz, M.: Predictive QoS-based admission control for multiclass traffic in cellular wireless networks. IEEE J. Select. Areas Commun. 18 (2000) $523-534$

10. Wu, S., Wong, K.Y.M., Li, B.: A dynamic call admission policy with precision QoS guarantee using stochastic control for mobile wireless networks. IEEE/ACM Trans. Networking 10 (2002) 257-271

11. Ghaderi, M., Boutaba, R.: Mobile effective bandwidth and its application to admission control in cellular packet networks. Technical Report CS-2004-61, School of Computer Science, University of Waterloo (2004)

12. Schwartz, M.: Broadband Integrated Networks. Prentice Hall, New Jersey, USA (1996)

13. Ghaderi, M., Boutaba, R.: Stochastic admission control for quality of service in wireless packet networks. Technical Report CS-2004-60, School of Computer Science, University of Waterloo (2004)

14. Eun, D.Y., Shroff, N.B.: A measurement-analytic approach for QoS estimation in a network based on the dominant time scale. IEEE/ACM Trans. Networking 11 (2003) 222-235

15. Jedrzycki, C., Leung, V.C.M.: Probability distribution of channel holding time in cellular telephone systems. In: Proc. IEEE VTC'96. Volume 1., Atlanta, GA (1996) 247-251 